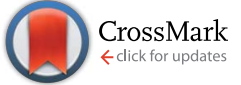

Cite this: J. Mater. Chem. A, 2015, 3, 7710

Received 11th December 2014

Accepted 5th March 2015

DOI: $10.1039 / c 4 t a 06818 a$

www.rsc.org/MaterialsA

\section{Improved hydrogen storage in Ca-decorated boron heterofullerenes: a theoretical study}

\author{
Süleyman Er, $\uparrow^{\text {*a }}$ Gilles A. de Wijs ${ }^{b}$ and Geert Brocks ${ }^{c}$
}

We computationally investigate the hydrogen storage properties of calcium-decorated $\mathrm{C}_{48} \mathrm{~B}_{12}$ boron-carbon heterofullerene molecules, and compare them to $\mathrm{C}_{60}$ (all-carbon) fullerene decorated with calcium. We employ density functional theory (DFT) on the lowest energy configurations of $\mathrm{C}_{48} \mathrm{~B}_{12}$ molecules and find that these molecules have the following properties. (1) The most stable $\mathrm{C}_{48} \mathrm{~B}_{12}$ isomers have an electron affinity that is $0.93-1.04 \mathrm{eV}$ higher than their carbon only counterpart. (2) The binding of a $\mathrm{Ca}$ atom to $\mathrm{C}_{48} \mathrm{~B}_{12}$ is $\sim 2.2 \mathrm{eV}$ stronger than its binding to $\mathrm{C}_{60}$. (3) Unlike $\mathrm{C}_{60} \mathrm{Ca}_{x} x=1-6, \mathrm{C}_{48} \mathrm{~B}_{12} \mathrm{Ca}_{x}$ is stable with respect to decomposition into the fullerene molecules and $\mathrm{Ca}$ bulk metal. (4) $\mathrm{C}_{48} \mathrm{~B}_{12} \mathrm{Ca}_{x}$ binds up to six hydrogen molecules per metal center, leading to a gravimetric density of up to 7.1 weight percent (wt\%). The hydrogen binding energies of up to $\sim 0.24 \mathrm{eV}$ open a prospect of hydrogen storage at ambient temperature.

Hydrogen is a clean energy carrier that shows promise in decreasing our dependence on fossil fuels. The US Department of Energy (DOE) suggests that by the year 2020, a successful onboard hydrogen storage system should have a hydrogen storage capacity of at least $5.5 \mathrm{wt} \%$ at moderate pressures, and should operate at delivery temperatures between -40 and $85{ }^{\circ}$ C. ${ }^{1}$ The speed of (un)loading of hydrogen (from)to the storage material should be at least such that $5 \mathrm{~kg}$ of hydrogen should be loaded within 200 seconds. ${ }^{1}$ Physisorption is preferred to chemisorption as no bonds need to be formed or broken during the process.

Physisorption materials with open or porous structures facilitate fast hydrogen kinetics, which facilitates loading of

\footnotetext{
${ }^{a}$ Department of Chemistry and Chemical Biology, Harvard University, 12 Oxford Street, Cambridge, MA 02138, USA. E-mail: ser@chemistry.harvard.edu

${ }^{b}$ Institute for Molecules and Materials, Radboud University, Heyendaalseweg 135, 6525 AJ Nijmegen, The Netherlands

${ }^{c}$ Faculty of Science and Technology and MESA+ Institute for Nanotechnology, University of Twente, P.O. Box 217, 7500 AE Enschede, The Netherlands

$\dagger$ Present address: Leiden Institute of Chemistry, Gorlaeus Laboratories, Leiden University, P.O. Box 9502, 2300 RA Leiden, The Netherlands. E-mail: s.er@ lic.leidenuniv.nl
}

hydrogen into the storage system. In physisorption materials, $\mathrm{H}_{2}$ molecules are adsorbed onto substrates, typically via weak, van der Waals type interactions. Thus, binding hydrogen molecules to host materials requires very low temperatures and high hydrogen gas pressures. Such conditions are generally unsuitable for mobile applications, which require safe and practical means of storing hydrogen.

To improve the binding of hydrogen to physisorption materials, recent research has focused on dispersing metal atoms onto these materials. A stronger interaction with hydrogen molecules has important consequences for thermodynamics, and increases the operating temperatures of storage materials. To restrict the increase of the total system weight, resulting from the addition of metals, only a limited number of metal atoms can be used to decorate lightweight hosts. Atoms of simple, electropositive alkali or alkaline earth metals form very polar bonds with carbon-based (or boron-based) materials. Electrostatic interactions between (partially charged) metal atoms and the induced dipoles or multipoles on hydrogen molecules result in a binding energy that is 2-3 times higher than a typical van der Waals bond between hydrogen and a carbon-based material. ${ }^{2,3}$ Alkaline earth metals, such as $\mathrm{Ca}$, have a larger effect on the binding energy than alkali metals, such as Li or Na. Recent studies have shown that functionalizing lightweight hosts with $\mathrm{Ca}$ atoms has important benefits. ${ }^{\mathbf{4 - 1 9}}$

Dispersing transition metal atoms, such as Sc or Ti, on carbon-based substrates, makes the adsorption energy of hydrogen molecules even larger. ${ }^{\mathbf{2 0 - 2 4}}$ However, not all hydrogens are then bonded equally. Upon loading, the first hydrogen molecules are strongly chemisorbed in atomic form, and the subsequent ones are physisorbed in molecular form. This means that the full storage capacity cannot be addressed at a single operating temperature. Moreover, clustering of metal atoms will almost certainly occur, because of the relatively large cohesive energy of these transition metals ( $\geq 4 \mathrm{eV}$ per atom). This, in turn, degrades the storage capacity even further. ${ }^{25} \mathrm{Ca}$ metal has a relatively small cohesive energy ( $\leq 2 \mathrm{eV}$ per atom), so $\mathrm{Ca}$ atoms have a lower tendency to cluster on the host material 
once they are deposited. Ca atoms are expected to bind all hydrogen molecules reversibly via physisorption. Moreover, $\mathrm{Ca}$ is an abundant element, which is relatively low-cost when compared with transition metals such as Sc or Ti.

The experimental and theoretical studies done so far mainly explored pure carbon-based systems (fullerenes, nanotubes, graphene, graphyne and carbyne). ${ }^{7-19}$ In this work, we study the Ca decoration of carbon-boron heterofullerenes. The first heterofullerenes realized in experiments were boron-doped carbon fullerenes. ${ }^{26,27}$ Since then, a variety of heterofullerenes with different boron concentrations have been produced on a macroscopic scale. ${ }^{28-37}$ We show that incorporating boron stabilizes the Ca-decorated fullerene, and also increases the interaction of hydrogen molecules with this system. The optimal interaction strength can be obtained from the rationalization that follows.

The adsorption of molecules, such as $\mathrm{H}_{2}$, under common conditions does not usually involve a first-order phase transition. This means that when operating at a fixed temperature $T$, the storing and unloading of hydrogen have to be done at two different gas pressures $P_{1}$ and $P_{2}$, respectively. Assuming that physisorption of hydrogen can be described by the Langmuir adsorption model, ${ }^{38}$ the fraction $f$ of the total adsorption capacity that is reversibly loaded and unloaded at these pressures is maximally

$$
f=\frac{\sqrt{P_{1} / P_{2}}-1}{\sqrt{P_{1} / P_{2}}+1},
$$

if the change in free energy $\Delta G_{0}$, which determines the adsorption reaction constant, is equal to

$$
\Delta G_{0}=\Delta H_{0}-T \Delta S_{0}=\frac{R T}{2} \ln \left[\frac{P_{1} P_{2}}{P_{0}^{2}}\right],
$$

where $P_{0}=1$ bar is the standard pressure. Using Monte Carlo simulations, Bhatia and Myers ${ }^{38}$ have argued that for hydrogen adsorption on a range of carbon substrates, the entropy change at standard pressure $\Delta S_{0} \approx-8 R$. It means that, e.g., at room temperature for $P_{1}=30 \mathrm{bar}$ and $P_{2}=1.5 \mathrm{bar}$, the optimum adsorption enthalpy is $\Delta H_{0} \approx-0.15 \mathrm{eV}$ per $\mathrm{H}_{2}$ molecule. As intrinsic properties, such as vibrations, of hydrogen molecules hardly change upon physisorption, one may approximate $\Delta H_{0}$ by the $T=0$ hydrogen binding energy, and neglect the contributions of zero-point motions. On substrates other than carbon based materials, a variation in the entropy change at standard pressure $\left(\Delta S_{0}\right)$ shifts the enthalpy change at standard pressure $\left(\Delta H_{0}\right)$. Recent experiments on porous solids with cationic metal centers, such as cation-exchanged zeolites and metal-organic frameworks with coordinatively unsaturated cationic metal atoms, suggest a positive and nonlinear correlation between $\Delta S_{0}$ and $\Delta H_{0}$. These new studies estimate the ideal value of hydrogen binding energies $\left(-\Delta H_{0}\right)$ to be between 0.23 and 0.26 $\mathrm{eV}$ per $\mathrm{H}_{2} \cdot{ }^{39,40}$ Accordingly, for the materials considered in the present work, we aim for molecular hydrogen binding energies that are between 0.15 and $0.26 \mathrm{eV}$ per $\mathrm{H}_{2}$.

We calculate such adsorption energies within density functional theory (DFT) calculations with the PW91 functional of the generalized gradient approximation (GGA), ${ }^{\mathbf{4 1}}$ using the Vienna $A b$ initio Simulation Package (VASP). ${ }^{\mathbf{4 2 - 4 4}}$ Projector augmented waves (PAW) $)^{\mathbf{4 5}, 46}$ are employed, treating, for B and C, the electrons in the 1s shell as the frozen core, and for Ca all electrons up to and including the $3 \mathrm{~s}$ shell. The PAW technique and the GGA-PW91 functional are sufficiently accurate for studying the interaction between molecular hydrogen and metals., ${ }^{2,3,647}$ To account for the dispersion (van der Waals) interactions we also carry out calculations using the DFT-D2 method of Grimme with its default force-field parameters as implemented in VASP. ${ }^{48}$ Grimme's empirical correction scheme for common density functionals has been applied successfully to many chemical problems including molecular hydrogen. ${ }^{\mathbf{4 , 4 9 - 5 4}}$ We find however that Ca loses part of its valence electrons. The resulting $\mathrm{Ca}$ ion is more difficult to polarize, hence its van der Waals interaction with molecular hydrogen is probably weakened. Thus, PW91 results can be taken as lower bounds for hydrogen binding energies whereas DFT-D2 results can be taken as upper bounds. The kinetic energy cutoff for the plane waves is set to $400 \mathrm{eV}$ and the conjugate gradient (CG) algorithm is used for the geometry optimizations. All molecules are optimized using a periodic cubic box and $\Gamma$-point sampling. A sufficiently large cell parameter of $30 \AA$ is used to avoid significant interactions between the periodic images. The optimizations are assumed to be complete when the total remaining forces on the atoms are lower than $0.01 \mathrm{eV} \AA^{-1}$. In the following, we report the calculated numbers using the DFT-D2 method of Grimme. When discussing small binding energies, where van der Waals interactions could be significant, we report both DFT-D2 and PW91 results.

We calculate the binding energy $E_{\mathrm{b}}^{\mathrm{Ca}}$ of a $\mathrm{Ca}$ atom to a (hetero)fullerene molecule from

$$
E_{\mathrm{b}}^{\mathrm{Ca}}=\frac{1}{x}\left[E_{\mathrm{C}_{48} \mathrm{~B}_{12}}+x E_{\mathrm{Ca}_{(\text {bulk })}}-E_{\mathrm{C}_{48} \mathrm{~B}_{12} \mathrm{Ca}_{x}}\right],
$$

where $E_{\mathrm{C}_{48} \mathrm{~B}_{12}}$ is the total energy of a (hetero)fullerene molecule, $E_{\mathrm{Ca}_{\text {(bulk) }}}$ is the total energy per atom of calcium bulk metal, and $E_{\mathrm{C}_{48} \mathrm{~B}_{12} \mathrm{Ca}_{x}}$ is the total energy of a (hetero)fullerene molecule decorated with $x \mathrm{Ca}$ atoms. Note that a positive value of $E_{\mathrm{b}}^{\mathrm{Ca}}$ means that the Ca-decorated (hetero)fullerene molecule is stable.

We first consider the interaction of a single $\mathrm{Ca}$ atom with a (hetero)fullerene molecule. We find that the Ca atom does not bind strongly to carbon-only fullerene. Using the total energy $E_{\mathrm{Ca}_{\text {(atom) }}}$ of an isolated Ca atom instead of the bulk energy $E_{\mathrm{Ca}_{\text {(bulk) }}}$ in eqn (3), we find a binding energy of $1.437 \mathrm{eV}$, in accordance with a previous study. ${ }^{19}$ However, if we use $E_{\mathrm{Ca}_{\text {(bulk) }}}$ as a reference energy, as is more appropriate, then $E_{\mathrm{b}}^{\mathrm{Ca}}=-0.666 \mathrm{eV}$. Such a negative value indicates that Ca-decorated $\mathrm{C}_{60}$ molecules are thermodynamically not stable against decomposition into $\mathrm{C}_{60}$ and $\mathrm{Ca}$ bulk. Therefore, there is an intrinsic driving force towards the clustering of $\mathrm{Ca}$ atoms, which severely impairs the potential use of $\mathrm{C}_{60} \mathrm{Ca}$ as a hydrogen storage material.

The binding between $\mathrm{Ca}$ and $\mathrm{C}_{60}$ is polar. If we perform a Bader charge analysis ${ }^{55,56}$ to estimate the effective charges on the atoms, we find that the $\mathrm{Ca}$ atom attains a charge of $+1.38 e$. To increase the binding between $\mathrm{Ca}$ and fullerene, we consider 
a)

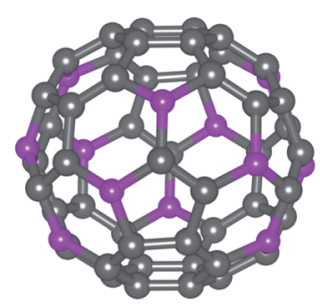

b)

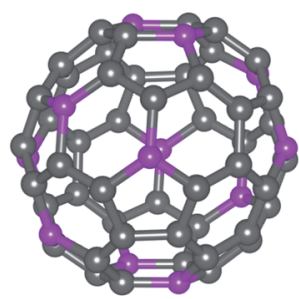

Fig. 1 Perspective views of DFT optimized $C_{48} B_{12}$ heterofullerenes: (a) the $S_{6}$ isomer, and (b) the $C_{i}$ isomer. Carbon and boron atoms are shown as grey and purple spheres, respectively.

substitution of fullerene carbons with boron atoms in the latter. This creates an electron-deficient molecule and hence a better acceptor than $\mathrm{C}_{60}$. Previous studies have shown that such substitutions are possible up to boron concentrations of 20 atom\%, while retaining the fullerene structure..$^{26,28-30,57-60}$ $\mathrm{C}_{48} \mathrm{~B}_{12}$ has two low energy isomers of $\mathrm{S}_{6}$ and $\mathrm{C}_{i}$ symmetry. ${ }^{57,58,60}$ We find that the $\mathrm{S}_{6}$ isomer is $0.836 \mathrm{eV}$ per molecule lower in energy that the $\mathrm{C}_{i}$ isomer, making the former thermodynamically much more stable. The increased acceptor character of these boron-substituted fullerenes is illustrated by their electron affinity. The calculated electron affinity of $\mathrm{C}_{60}$ is $2.77 \mathrm{eV}$, in good agreement with the earlier theoretical ${ }^{57}(2.40 \mathrm{eV})$ and the experimental $^{61}(2.69 \mathrm{eV})$ results. The calculated electron affinities for the $\mathrm{S}_{6}$ and $\mathrm{C}_{i}$ isomers of $\mathrm{C}_{48} \mathrm{~B}_{12}$ are $3.70 \mathrm{eV}$ and $3.81 \mathrm{eV}$, respectively.

Both the $\mathrm{C}_{48} \mathrm{~B}_{12}$ isomers have pentagons containing a single $B$ atom (Fig. 1). The $S_{6}$ isomer contains eight all-carbon and twelve $\mathrm{C}_{4} \mathrm{~B}_{2}$ hexagons (Fig. 1a). Six of the latter contain B in para positions, whereas the others contain $\mathrm{B}$ in meta positions. The $\mathrm{C}_{i}$ isomer contains two all-carbon, twelve $\mathrm{C}_{5} \mathrm{~B}_{1}$, and six $\mathrm{C}_{4} \mathrm{~B}_{2}$ hexagons (Fig. 1b). All six of the latter have $\mathrm{B}$ in para positions.

We find that the most favorable binding site of $\mathrm{Ca}$ is over the $\mathrm{C}_{4} \mathrm{~B}_{2}$ hexagon centers, both in the $\mathrm{S}_{6}$ and the $\mathrm{C}_{i}$ isomers, as shown in Fig. 2. The calculated binding energies of a single $\mathrm{Ca}$ atom, $x=1$ in eqn (3), are $1.564 \mathrm{eV}$ and $1.575 \mathrm{eV}$ to the $\mathrm{S}_{6}$ and $\mathrm{C}_{i}$ isomers, respectively. These values clearly demonstrate that binding of $\mathrm{Ca}$ to $\mathrm{C}_{48} \mathrm{~B}_{12}$ is much stronger than to $\mathrm{C}_{60}$. In contrast to $\mathrm{C}_{60} \mathrm{Ca}, \mathrm{C}_{48} \mathrm{~B}_{12} \mathrm{Ca}$ is stable with respect to clustering of $\mathrm{Ca}$ atoms, i.e. separation into $\mathrm{Ca}$ bulk and $\mathrm{C}_{48} \mathrm{~B}_{12}$ molecules. Similar to $\mathrm{C}_{60} \mathrm{Ca}$, we perform a Bader charge analysis on $\mathrm{C}_{48} \mathrm{~B}_{12} \mathrm{Ca}$. The calculated Bader charges on $\mathrm{Ca}$ are $+1.52 e$ and to $+1.51 e$ for the $\mathrm{S}_{6}$ and $\mathrm{C}_{i}$ isomers, respectively, indicating that the

bonding has a substantial ionic character, and that the two isomers behave very similarly regarding their bonding to $\mathrm{Ca}$.

Next, we study the adsorption of $\mathrm{H}_{2}$ molecules on $\mathrm{C}_{48} \mathrm{~B}_{12} \mathrm{Ca}$. We calculate the binding energy of hydrogen molecules to $\mathrm{C}_{48} \mathrm{~B}_{12} \mathrm{Ca}_{x}$ using eqn (4).

$$
E_{\mathrm{b}}^{\mathrm{H}_{2}}=\frac{1}{y}\left[E_{\mathrm{C}_{48} \mathrm{~B}_{12} \mathrm{Ca}_{x}}+y E_{\mathrm{H}_{2}}-E_{\mathrm{C}_{48} \mathrm{~B}_{12} \mathrm{Ca}_{x}\left(\mathrm{H}_{2}\right)_{y}}\right],
$$

where $E_{\mathrm{H}_{2}}$ and $E_{\mathrm{C}_{48} \mathrm{~B}_{22} \mathrm{Ca}_{x}\left(\mathrm{H}_{2}\right)_{y}}$ are the total energies of the hydrogen molecule and the stepwise hydrogenated (hetero) fullerene-Ca system, respectively. A single $\mathrm{Ca}$ atom $(x=1)$ can bind up to six $\mathrm{H}_{2}$ molecules, as illustrated in Fig. 2, with five $\mathrm{H}_{2}$ in a ring around the protruding $\mathrm{Ca}$ atom, and the sixth $\mathrm{H}_{2}$ molecule on top of the $\mathrm{Ca}$ atom. The first $\mathrm{H}_{2}$ molecule is bonded with an energy of $0.265 \mathrm{eV}$ per $\mathrm{H}_{2}$ and $0.243 \mathrm{eV}$ per $\mathrm{H}_{2}$, to the Cadecorated $\mathrm{S}_{6}$ and $\mathrm{C}_{i}$ isomers of $\mathrm{C}_{48} \mathrm{~B}_{12}$, respectively. Additional $\mathrm{H}_{2}$ molecules bind somewhat less strongly, see Table 1 . At their fully hydrogenated states, the Ca-decorated $\mathrm{S}_{6}$ and $\mathrm{C}_{i}$ isomers bind the hydrogen molecules with average binding energies of $0.245 \mathrm{eV}$ per $\mathrm{H}_{2}$ and $0.242 \mathrm{eV}$ per $\mathrm{H}_{2}$, respectively. The calculations using the PW91 functional give similar results to the DFTD2 method, but the average hydrogen binding energies are approximately $0.1 \mathrm{eV}$ lower for both of the isomers (Table 1).

As $\mathrm{C}_{48} \mathrm{~B}_{12}$ is isoelectronic with $\mathrm{C}_{60}^{12+}$, and the bonding with $\mathrm{Ca}$ atoms has a substantial ionic character, one expects that $\mathrm{C}_{48} \mathrm{~B}_{12}$ accommodates six $\mathrm{Ca}$ atoms with similar binding characteristics. This is achieved by putting a $\mathrm{Ca}$ atom over the centers of all $\mathrm{C}_{4} \mathrm{~B}_{2}$ hexagons, both in the $\mathrm{S}_{6}$ and the $\mathrm{C}_{i}$ isomers. Each of these $\mathrm{Ca}$ atoms is bonded strongly to $\mathrm{C}_{48} \mathrm{~B}_{12}$ with average binding energies of $1.046 \mathrm{eV}$ per atom and $1.082 \mathrm{eV}$ per atom for the $\mathrm{S}_{6}$ and $\mathrm{C}_{i}$ isomers, respectively (eqn. (3)). Note that, since these binding energies are calculated with respect to $\mathrm{Ca}$ bulk, we expect $\mathrm{C}_{48} \mathrm{~B}_{12} \mathrm{Ca}_{6}$ to be stable against the clustering of $\mathrm{Ca}$ atoms, which is again in contrast to Ca-decorated $\mathrm{C}_{60}$.

The optimized structures of the fully hydrogenated $\mathrm{C}_{48} \mathrm{~B}_{12} \mathrm{Ca}_{6}$ molecules are shown in Fig. 3. As for $\mathrm{C}_{48} \mathrm{~B}_{12} \mathrm{Ca}$, we find that a maximum of six $\mathrm{H}_{2}$ molecules are adsorbed per $\mathrm{Ca}$ atom. Consequently, the fully hydrogenated molecules have the chemical formula $\mathrm{C}_{48} \mathrm{~B}_{12} \mathrm{Ca}_{6}\left(\mathrm{H}_{2}\right)_{36}$. Each heterofullerene molecule then contains $7.1 \mathrm{wt} \% \mathrm{H}_{2}$ with average binding energies of $0.249 \mathrm{eV}$ per $\mathrm{H}_{2}\left(0.152 \mathrm{eV}\right.$ per $\mathrm{H}_{2}$ with PW91) and $0.244 \mathrm{eV}$ per $\mathrm{H}_{2}$ (0.148 eV per $\mathrm{H}_{2}$ with PW91), for the $\mathrm{S}_{6}$ and $\mathrm{C}_{i}$ isomers, respectively.

For physisorbed molecules, one may expect that these energies also determine the kinetics, and that no additional
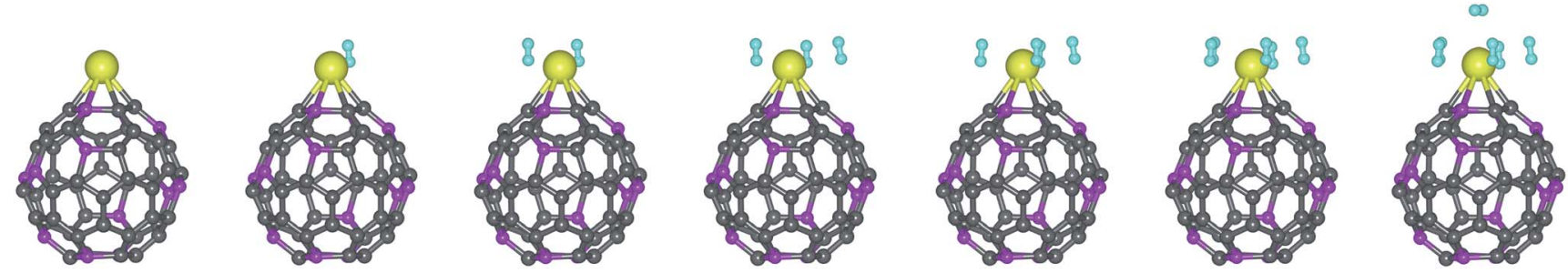

Fig. 2 Optimized structures of $\mathrm{C}_{48} \mathrm{~B}_{12} \mathrm{Ca}\left(\mathrm{H}_{2}\right)_{y}, y=1-6$ of the $\mathrm{S}_{6}$ isomer. Carbon, boron, calcium and hydrogen atoms are shown as grey, purple, yellow and turquoise spheres, respectively. 
Table 1 Calculated binding energies of $\mathrm{Ca}$ atoms to $\mathrm{C}_{48} \mathrm{~B}_{12}$ isomers with respect to bulk Ca metal $\left(E_{\mathrm{b}}^{\mathrm{Ca}}\right)$, Bader charges on the $\mathrm{Ca}$ atoms $(Q)$, average binding energies $\left(E_{b}^{\mathrm{H}_{2}}\right)$ of $\mathrm{H}_{2}$ molecules to $\mathrm{C}_{48} \mathrm{~B}_{12} \mathrm{Ca}$ (see Fig. 2)

\begin{tabular}{llllllllll}
\hline & \multicolumn{8}{c}{$E_{\mathrm{b}}^{\mathrm{H}_{2}}(\mathrm{eV})$} \\
\cline { 5 - 10 } Isomer & $E_{\mathrm{b}}^{\mathrm{Ca}}(\mathrm{eV})$ & $Q(\mathrm{e})$ & $1 \mathrm{H}_{2}$ & $2 \mathrm{H}_{2}$ & $3 \mathrm{H}_{2}$ & $4 \mathrm{H}_{2}$ & $5 \mathrm{H}_{2}$ & $6 \mathrm{H}_{2}$ \\
\hline $\mathrm{S}_{6}$ & 1.616 & +1.47 & 0.175 & 0.163 & 0.164 & 0.156 & 0.151 & 0.152 \\
$\mathrm{~S}_{6}$ (DFT-D2) & 1.564 & +1.52 & 0.265 & 0.255 & 0.255 & 0.249 & 0.246 & 0.245 \\
$\mathrm{C}_{i}$ & 1.626 & +1.46 & 0.161 & 0.161 & 0.154 & 0.156 & 0.149 & 0.149 \\
$\mathrm{C}_{i}$ (DFT-D2) & 1.575 & +1.51 & 0.243 & 0.243 & 0.239 & 0.246 & 0.243 & 0.242 \\
& & & & & & & & \\
\hline
\end{tabular}

kinetic energy barriers are involved in the adsorption process. To illustrate this, we carried out $a b$ initio molecular dynamics (IAMD) simulations on the $\mathrm{C}_{48} \mathrm{~B}_{12} \mathrm{Ca}\left(\mathrm{H}_{2}\right)_{6}$ molecule in the microcanonical ensemble. ${ }^{62}$ Due to the presence of lightweight elements, we used a short time step of $1.0 \mathrm{fs}$ in the AIMD simulations. Using the average kinetic energy of the atoms as a measure of the temperature, the latter is stepwise increased from $50 \mathrm{~K}$ to $300 \mathrm{~K}$ in $50 \mathrm{~K}$ steps, where at each temperature the simulations are performed for 15 ps. Fig. 4 shows the radial distribution function for $\mathrm{Ca}-\mathrm{H}$ pairs and the structures at the end of each equilibration step. In the simulation run, five hydrogen molecules stay adsorbed at temperatures $\leq 150 \mathrm{~K}$, and all are desorbed at temperatures $\geq 300 \mathrm{~K}$. This behavior is consistent with the absence of any kinetic barrier for desorption.

a)

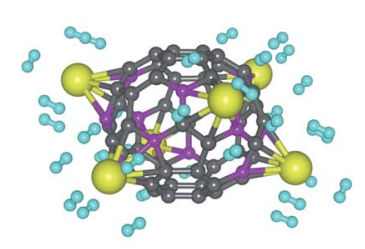

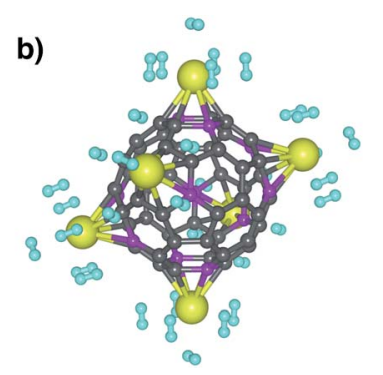

Fig. 3 Perspective views of the fully optimized Ca-decorated heterofullerenes in their fully hydrogenated states, $\mathrm{C}_{48} \mathrm{~B}_{12} \mathrm{Ca}_{6}\left(\mathrm{H}_{2}\right)_{36}$; ( $\mathrm{a}$ and b) correspond to the $S_{6}$ and $C_{i}$ isomers, respectively.

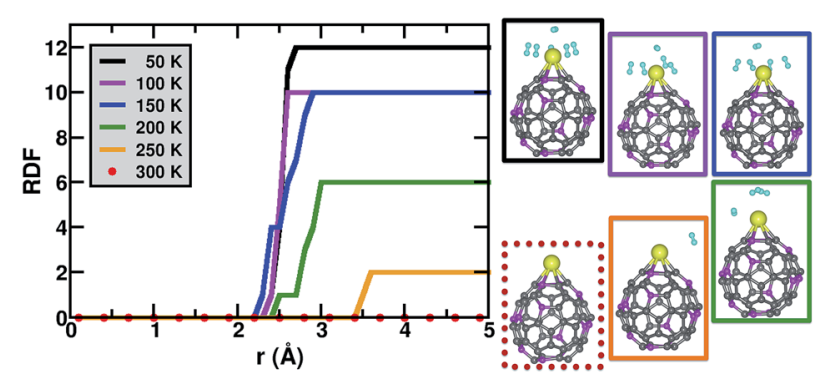

Fig. 4 The radial distribution functions (RDFs) of $\mathrm{Ca}-\mathrm{H}$ at the end of 15 ps AIMD equilibration runs using the DFT-D2 method at 50, 100, $150,200,250$ and $300 \mathrm{~K}$ for the $\mathrm{S}_{6}$ isomer based $\mathrm{C}_{48} \mathrm{~B}_{12} \mathrm{Ca}\left(\mathrm{H}_{2}\right)_{6}$ molecule. The boxes shown at the right show the structures at the end of each run.
In summary, we studied the functionalization of boron heterofullerenes with $\mathrm{Ca}$ atoms for high density hydrogen storage. We find that six Ca atoms interact strongly with $\mathrm{C}_{48} \mathrm{~B}_{12}$, and the dispersed $\mathrm{Ca}$ atoms are thermodynamically stable against metal clustering, unlike $\mathrm{Ca}$ atoms dispersed on $\mathrm{C}_{60}$. The bonding between $\mathrm{Ca}$ and $\mathrm{C}_{48} \mathrm{~B}_{12}$ has a substantial ionic character, and the well-exposed, (partially) charged Ca atoms bind up to six $\mathrm{H}_{2}$ molecules per Ca with an average binding energy of $\sim 0.24 \mathrm{eV}$ per $\mathrm{H}_{2}$ (DFT-D2) and $\sim 0.15 \mathrm{eV}$ per $\mathrm{H}_{2}$ (PW91). These energies are in the range needed for onboard hydrogen storage, yielding $7.1 \mathrm{wt} \% \mathrm{H}_{2}$. Furthermore, the predicted hydrogen binding energies are only weakly dependent on $\mathrm{H}_{2}$ loading.

\section{Acknowledgements}

S. E. performed work as part of the Fellowships for Young Energy Scientists program of the Foundation for Fundamental Research on Matter (FOM), which is part of the Netherlands Organization for Scientific Research (NWO). The computing time was provided by the Center for Functional Nanomaterials, Brookhaven National Laboratory, which is supported by the U.S. Department of Energy, Office of Basic Energy Sciences, under Contract no. DE-AC02-98CH10886. We thank A. Aspuru-Guzik for helpful discussions.

\section{References}

1 US Department of Energy Fuel Cell Technologies Office Multi-Year Research, Development and Demonstration Plan, http://energy.gov/sites/prod/files/2014/11/f19/fcto_myrdd_ storage.pdf, accessed: 2015-03-11.

2 S. Er, G. A. de Wijs and G. Brocks, J. Phys. Chem. C, 2009, 113, 8997-9002.

3 S. Er, G. A. de Wijs and G. Brocks, J. Phys. Chem. C, 2009, 113, 18962-18967.

4 X. Chen, F. Yuan, Q. Gu and X. Yu, J. Mater. Chem. A, 2013, 1, 11705.

5 F. Gao, Z. Ding and S. Meng, Sci. Rep., 2013, 3, 1882.

6 N. Park, K. Choi, J. Hwang, D. W. Kim, D. O. Kim and J. Ihm, Proc. Natl. Acad. Sci. U. S. A., 2012, 109, 19893-19899.

7 T. Hussain, B. Pathak, M. Ramzan, T. A. Maark and R. Ahuja, Appl. Phys. Lett., 2012, 100, 183902.

8 P. B. Sorokin, H. Lee, L. Y. Antipina, A. K. Singh and B. I. Yakobson, Nano Lett., 2011, 11, 2660-2665.

9 C. Li, J. Li, F. Wu, S.-S. Li, J.-B. Xia and L.-W. Wang, J. Phys. Chem. C, 2011, 115, 23221-23225.

10 C. Cazorla, S. A. Shevlin and Z. X. Guo, Phys. Rev. B: Condens. Matter Mater. Phys., 2010, 82, 155454.

11 Q. Wang, Q. Sun, P. Jena and Y. Kawazoe, J. Chem. Theory Comput., 2009, 5, 374-379.

12 G. Wu, J. Wang, X. Zhang and L. Zhu, J. Phys. Chem. C, 2009, 113, 7052-7057.

13 G. Kim and S.-H. Jhi, J. Phys. Chem. C, 2009, 113, 2049920503.

14 M. Li, Y. Li, Z. Zhou, P. Shen and Z. Chen, Nano Lett., 2009, 9, 1944-1948. 
15 H. Lee, J. Ihm, M. L. Cohen and S. G. Louie, Phys. Rev. B: Condens. Matter Mater. Phys., 2009, 80, 115412.

16 H. Lee, J. Ihm, M. L. Cohen and S. G. Louie, Nano Lett., 2010, 10, 793-798.

17 X. Yang, R. Q. Zhang and J. Ni, Phys. Rev. B: Condens. Matter Mater. Phys., 2009, 79, 075431.

18 G. Kim, S.-H. Jhi, S. Lim and N. Park, Phys. Rev. B: Condens. Matter Mater. Phys., 2009, 79, 155437.

19 M. Yoon, S. Yang, C. Hicke, E. Wang, D. Geohegan and Z. Zhang, Phys. Rev. Lett., 2008, 100, 206806.

20 T. Yildirim and S. Ciraci, Phys. Rev. Lett., 2005, 94, 175501.

21 Y. Zhao, Y.-H. Kim, A. C. Dillon, M. J. Heben and S. B. Zhang, Phys. Rev. Lett., 2005, 94, 155504.

22 H. Lee, W. I. Choi and J. Ihm, Phys. Rev. Lett., 2006, 97, 056104 .

23 G. Kim, S.-H. Jhi and N. Park, Appl. Phys. Lett., 2008, 92, 013106.

24 E. Durgun, S. Ciraci and T. Yildirim, Phys. Rev. B: Condens. Matter Mater. Phys., 2008, 77, 085405.

25 Q. Sun, Q. Wang, P. Jena and Y. Kawazoe, J. Am. Chem. Soc., 2005, 127, 14582-14583.

26 T. Guo, C. Jin and R. E. Smalley, J. Phys. Chem., 1991, 95, 4948-4950.

27 Y. Chai, T. Guo, C. Jin, R. E. Haufler, L. P. F. Chibante, J. Fure, L. Wang, J. M. Alford and R. E. Smalley, J. Phys. Chem., 1991, 95, 7564-7568.

28 D. Golberg, Y. Bando, K. Kurashima and T. Sasaki, Appl. Phys. Lett., 1998, 72, 2108.

29 D. Golberg, Y. Bando, O. Stéphan, L. Bourgeois, K. Kurashima, T. Sasaki, T. Sato and C. Goringe, J. Electron Microsc., 1999, 48, 701-709.

30 Y. J. Zou, X. W. Zhang, Y. L. Li, B. Wang, H. Yan, J. Z. Cui, L. M. Liu and D. A. Da, J. Mater. Sci., 2002, 37, 1043-1047.

31 H.-J. Muhr, R. Nesper, B. Schnyder and R. Kötz, Chem. Phys. Lett., 1996, 249, 399-405.

32 B. Cao, X. Zhou, Z. Shi, Z. Gu, H. Xiao and J. Wang, Fullerene Sci. Technol., 1998, 6, 639-648.

33 H. Lange, A. Huczko, P. Byszewski, E. Mizera and H. Shinohara, Chem. Phys. Lett., 1998, 289, 174-180.

34 T. Nakamura, K. Ishikawa, K. Yamamoto, T. Ohana, S. Fujiwara and Y. Koga, Phys. Chem. Chem. Phys., 1999, 1, 2631-2633.

35 J. C. Hummelen, C. Bellavia-Lund and F. Wudl, Fullerenes and Related Structures, Springer Verlag, Berlin Heidelberg, 1999, vol. 199, pp. 93-134.

36 A. A. Arie, B.-J. Jeon and J.-K. Lee, Carbon Lett., 2010, 11, 127130.

37 P. W. Dunk, A. Rodríguez-Fortea, N. K. Kaiser, H. Shinohara, J. M. Poblet and H. W. Kroto, Angew. Chem., Int. Ed., 2012, 52, 315-319.
38 S. K. Bhatia and A. L. Myers, Langmuir, 2006, 22, 1688-1700. 39 E. Garrone, B. Bonelli and C. O. Areán, Chem. Phys. Lett., 2008, 456, 68-70.

40 C. O. Areán, S. Chavan, C. P. Cabello, E. Garrone and G. T. Palomino, ChemPhysChem, 2010, 11, 3237-3242.

41 J. P. Perdew and Y. Wang, Phys. Rev. B: Condens. Matter Mater. Phys., 1992, 45, 13244-13249.

42 G. Kresse and J. Hafner, Phys. Rev. B: Condens. Matter Mater. Phys., 1993, 47, 558-561.

43 G. Kresse and J. Furthmüller, Phys. Rev. B: Condens. Matter Mater. Phys., 1996, 54, 11169-11186.

44 G. Kresse and J. Furthmüller, Comput. Mater. Sci., 1996, 6, 15-50.

45 P. E. Blöchl, Phys. Rev. B: Condens. Matter Mater. Phys., 1994, 50, 17953-17979.

46 G. Kresse and D. Joubert, Phys. Rev. B: Condens. Matter Mater. Phys., 1999, 59, 1758-1775.

47 Y. Y. Sun, K. Lee, L. Wang, Y.-H. Kim, W. Chen, Z. Chen and S. B. Zhang, Phys. Rev. B: Condens. Matter Mater. Phys., 2010, 82, 073401.

48 S. Grimme, J. Comput. Chem., 2006, 27, 1787-1799.

49 M. P. Waller, H. Kruse, C. Mück-Lichtenfeld and S. Grimme, Chem. Soc. Rev., 2012, 41, 3119-3128.

50 C. Li, J. Li, F. Wu, S.-S. Li, J.-B. Xia and L.-W. Wang, J. Phys. Chem. C, 2011, 115, 23221-23225.

51 S. Lee, M. Lee, H. Choi, D. S. Yoo and Y.-C. Chung, Int. J. Hydrogen Energy, 2013, 38, 4611-4617.

52 Y. Wang, F. Wang, B. Xu, J. Zhang, Q. Sun and Y. Jia, J. Appl. Phys., 2013, 113, 064309.

53 Y. Wang, X. Li, F. Wang, B. Xu, J. Zhang, Q. Sun and Y. Jia, Chem. Phys., 2013, 415, 26-30.

54 S. Y. Willow and S. S. Xantheas, Chem. Phys. Lett., 2012, 525526, 13-18.

55 G. Henkelman, A. Arnaldsson and H. Jónsson, Comput. Mater. Sci., 2006, 36, 354-360.

56 W. Tang, E. Sanville and E. Henkelman, J. Phys.: Condens. Matter, 2009, 21, 084204.

57 R.-H. Xie, G. W. Bryant, J. Zhao, V. H. Smith Jr, A. D. Carlo and A. Pecchia, Phys. Rev. Lett., 2003, 90, 206602.

58 M. R. Manaa, Chem. Phys. Lett., 2003, 382, 194-197.

59 Y.-H. Kim, Y. Zhao, A. Williamson, M. J. Heben and S. B. Zhang, Phys. Rev. Lett., 2006, 96, 016102.

60 Q. Sun, Q. Wang and P. Jena, Appl. Phys. Lett., 2009, 94, 013111.

61 X.-B. Wang, H.-K. Woo and L.-S. Wang, J. Chem. Phys., 2005, 123, 051106.

62 D. Marx and J. Hutter, Ab Initio Molecular Dynamics: Theory and Implementation, John von Neumann Institute for Computing, Jülich, 2000, vol. 3, pp. 329-477. 